\title{
Panorama da Utilização de Jogos Digitais no Ensino de Programação no Nível Superior na Última Década: Uma Revisão Sistemática da Literatura
}

\author{
Rodrigo Ribeiro Silva ${ }^{1}$, Juliana Costa Fernandes ${ }^{1,2}$, Rodrigo Pereira dos Santos ${ }^{1}$ \\ ${ }^{1}$ Departamento de Informática Aplicada \& Programa de Pós-Graduação em Informática \\ Universidade Federal do Estado do Rio de Janeiro (UNIRIO), Brasil \\ ${ }^{2}$ Coordenação do Curso de Informática, Instituto Federal do Piauí (IFPI), Brasil \\ \{rodrigo.ribeiro,juliana.costa,rps\}@uniriotec.br
}

\begin{abstract}
The teaching of programming is part of the basic academic training in Computer Science and related areas. Several techniques and tools have been developed to support the teaching-learning process of programming, such as digital games. This paper aims to replicate a systematic literature review (SLR) covering 2008-2012, and present a new SLR regarding the use of digital games focused on teaching programming for beginners in Informatics at the higher education in Brazil covering 2013-2017. We compared the SLRs' results in the last decade and identified which tools and pedagogical theories are applied in teaching-learning of programming.
\end{abstract}

Resumo. O ensino de programação faz parte da formação acadêmica básica em Computação e áreas afins. Diversas técnicas e ferramentas são concebidas para apoiar o ensino-aprendizagem de disciplinas de programação, como a utilização de jogos digitais. Este artigo tem como objetivo replicar uma revisão sistemática da literatura (RSL) referente a 2008-2012, e apresentar uma nova RSL sobre a utilização de jogos digitais focada no ensino de programação para iniciantes em Computação no nível superior no Brasil, abrangendo 2013-2017. Foram comparados os resultados obtidos em ambos os períodos da última década e identificadas quais ferramentas e teorias pedagógicas são aplicadas para o ensino-aprendizagem de programação.

\section{Introdução}

Segundo as Diretrizes Curriculares Nacionais ${ }^{1}$, a disciplina de programação faz parte da formação básica em Computação e, à medida que a tecnologia evolui, a demanda por profissionais nessa área também cresce. Porém, um em cada três alunos ingressantes em Sistemas de Informação terminam o curso, enquanto que para o curso de Ciência da Computação, os números são de um para cada quatro ${ }^{2}$. Pesquisas apontam dificuldades dos estudantes, sendo a falta de compreensão do raciocínio lógico uma das principais razões pelo alto índice de reprovação e pela desistência de cursos de graduação da área [Santos et al. 2008]. Assim, muitas técnicas têm sido desenvolvidas para diminuir a evasão dos alunos. Uma delas é a gamificação que, para Kapp (2012) e Domínguez et al. (2013), significa o uso de jogos eletrônicos em contextos não relacionados a jogos.

\footnotetext{
${ }^{1}$ http://portal.mec.gov.br/index.php?option=com_docman\&view=download\&alias=52101-rces005-16pdf\&category_slug=novembro-2016-pdf\&Itemid $=30192$

${ }^{2} \mathrm{http} / /$ www.semesp.org.br/imprensa/indice-de-evasao-de-alunos-e-maior-na-area-de-tecnologia-dainformacao-2/
} 
VII Congresso Brasileiro de Informática na Educação (CBIE 2018)

Anais do XXIX Simpósio Brasileiro de Informática na Educação (SBIE 2018)

A fim de sedimentar as novas metodologias e ferramentas educacionais voltadas para o ensino-aprendizado de programação no cenário brasileiro, é importante mapear esforços e resultados de produções científicas nacionais. Este artigo apresenta então os resultados de uma revisão sistemática da literatura (RSL), que replica o trabalho de Medeiros et al. (2013), referente aos anos 2008 a 2012. Dessa forma, o objetivo é analisar os artigos sobre o uso de jogos digitais para o ensino de programação aos iniciantes em Computação no Brasil no nível superior, abrangendo os anos 2013 a 2017. Foram comparados os resultados obtidos na última década e identificadas ferramentas e teorias pedagógicas que são aplicadas para o ensino-aprendizagem de programação.

O restante do trabalho está organizado da seguinte forma: a Seção 2 apresenta a fundamentação teórica; na Seção 3, são apresentados os trabalhos relacionados; a Seção 4 apresenta o protocolo utilizado na RSL realizada; nas Seções 5 e 6, os resultados são apresentados e discutidos, respectivamente; por fim, a Seção 7 conclui o artigo.

\section{Fundamentação Teórica}

O modelo instrucionista é uma das metodologias de ensino mais utilizadas, em que o aluno assume um papel passivo na aquisição do conhecimento. O modelo construtivista, por sua vez, foca na aprendizagem, ou seja, o aluno se torna o construtor da própria sabedoria, tendo o professor como um agente facilitador do desenvolvimento cognitivo. No mundo globalizado, as pessoas estão acostumadas a acessarem informações de maneira rápida, direta e prática, de modo que os métodos de ensino vigentes podem tornar as salas de aula lugares entediantes. Para Piteira e Haddad (2011), isso gera baixa motivação entre os alunos e, segundo Scaico e Scaico (2016), o interesse é um aspecto que influencia no seu engajamento para aprender. Nesse contexto, os jogos educacionais surgem como alternativa, pois possibilitam um processo de aprendizagem participativo, comunicativo e capaz de levar o aprendiz a resolver problemas de forma lúdica.

Segundo Victal et al. (2015), os jogos digitais são importantes ferramentas para o aprendizado, justificando o seu uso na educação. Nesse sentido, Hoelfmann (2016) realizou um estudo que indica como os jogos podem auxiliar os alunos em aumentar a sua percepção, tendo um grande potencial para se tornarem importantes instrumentos no processo de ensino e aprendizagem. Para Uzunca e Jansen (2016), os jogos são uma nova forma de aprender, pois proporcionam: (1) o aprendizado em uma realidade virtual e/ou com papel simulado, o que encoraja o jogador, pois ele sempre pode recomeçar; (2) 'aprender fazendo', envolvendo-se, motivando-se e entretendo-se; (3) possibilitar a capacidade de encontrar e usar a informação sem a necessidade de memorização; e (4) simular ambientes complexos/caros e situações perigosas/críticas. A Tabela 1 mostra uma justificativa para o uso de jogos como forma de aprendizado.

Tabela 1. Justificativa para o uso de jogos. Fonte: [Uzunca e Jansen 2016]

\begin{tabular}{|c|l|}
\hline Justificativa & \multicolumn{1}{c|}{ Descrição } \\
\hline $\begin{array}{c}\text { Importância } \\
\text { crescente }\end{array}$ & Design, engenharia e produção de jogos sérios ainda estão em sua infância. \\
\hline $\begin{array}{c}\text { Alvo em } \\
\text { movimento }\end{array}$ & $\begin{array}{l}\text { Negócios emergentes com múltiplos usos nos setores da indústria, educação, saúde e } \\
\text { administração pública. }\end{array}$ \\
\hline $\begin{array}{c}\text { Potencial de } \\
\text { crescimento }\end{array}$ & 2,35 bilhões de euros no mercado global (estimativa do IDATE 2014 ${ }^{3}$ ). \\
\cline { 2 - 2 } & Mercado fragmentado e precisa de massa crítica para competir globalmente. \\
\hline
\end{tabular}

\footnotetext{
${ }^{3}$ http://www.idate.org/en/News/Serious-Games_643.html
} 
VII Congresso Brasileiro de Informática na Educação (CBIE 2018)

Anais do XXIX Simpósio Brasileiro de Informática na Educação (SBIE 2018)

\section{Trabalhos Relacionados}

Silva et al. (2014) investigaram publicações internacionais para realizar uma RSL sobre a eficácia do uso de jogos digitais para auxiliar o ensino-aprendizagem de programação. Os autores utilizaram as bases IEEEXplorer, ACM Digital Library e ScienceDirect (automatizada) e exploraram os anais dos eventos de Jogos Digitais e Informática na Educação (manual). A investigação ratificou a eficácia do uso dos jogos como ferramenta de ensino de programação. Scaico e Scaico (2016) realizaram uma RSL para identificar e analisar os estudos sobre o uso de jogos digitais em disciplinas introdutórias de programação em cursos superiores de Computação. $\mathrm{O}$ artigo também utilizou as bases IEEEXplorer, ACM Digital Library e ScienceDirect, além de procurar no Springer Link e CiteSeerX. Como no trabalho anterior, o foco foi buscar e catalogar publicações internacionais. Os resultados mostram que o uso de jogos no ensino introdutório de programação afeta positivamente o aprendizado e o engajamento.

Tendo em vista os trabalhos relacionados e a necessidade de investigar sobre utilização de jogos digitais no auxílio do ensino de programação para iniciantes em Computação no nível superior, apresentamos o panorama do cenário brasileiro na última década, considerando artigos publicados nos principais eventos e revistas nacionais.

\section{O Processo da Revisão Sistemática}

A revisão conduzida foi baseada no processo de Kitchenham e Charters (2007), em que uma abordagem sistemática é pré-definida por meio de um protocolo e métodos para identificar, avaliar e interpretar as evidências disponíveis, relacionadas a uma ou mais questões de pesquisa. O processo desta RSL é descrito nas próximas subseções.

\subsection{Questões de Pesquisa}

A definição das questões de pesquisa foi feita a partir dos critérios especificados pela estrutura PICO (população, intervenção, comparação e resultados) de Kitchenham e Charters (2007). Entretanto, uma parte dos atributos de PICO é considerada neste trabalho, como mostra a Tabela 2: população, intervenção e resultado (PIO). O motivo está no fato de que a meta é discutir o uso de jogos digitais no ensino de programação aos iniciantes em Computação no nível superior, e não os compará-los.

Seguindo a abordagem GQM (Goal-Question-Metric) [Basili 1992], o objetivo deste estudo foi formalizado: analisar o uso de jogos digitais no ensino de programação para iniciantes em Computação no nível superior com o propósito de caracterizar com respeito ao panorama da última década do ponto de vista de pesquisadores no contexto do cenário brasileiro. A partir desse objetivo, a questão de pesquisa (QP) desta RSL é: (QP) Qual o panorama do uso de jogos digitais no ensino de programação para iniciantes em Computação no nível superior na última década?. Para respondê-la, foram utilizadas subquestões de pesquisa (Sub-Q) propostas em Medeiros et al. (2013):

- Sub-Q1: Como os jogos digitais têm sido aplicados no ensino de programação?

- Sub-Q2: Que ferramentas têm sido empregadas?

- Sub-Q3: Quais os efeitos observados nos alunos com a introdução de atividades de programação baseadas em jogos digitais?

- Sub-Q4: Que teorias pedagógicas estão sendo utilizadas?

- Sub-Q5: Quais são as instituições de pesquisas envolvidas na área e como elas estão distribuídas no Brasil? 
VII Congresso Brasileiro de Informática na Educação (CBIE 2018)

Anais do XXIX Simpósio Brasileiro de Informática na Educação (SBIE 2018)

Tabela 2. Uso dos critérios da estrutura PIO

\begin{tabular}{|c|c|}
\hline População & Iniciantes em Computação no nível superior \\
\hline Intervenção & O uso de jogos digitais no ensino de programação \\
\hline Resultados & Panorama da última década \\
\hline
\end{tabular}

A SubQ de Medeiros et al. (2013) identificada como 'QP2' foi desconsiderada porque diz respeito ao público-alvo (nível médio e superior) e, em nosso estudo, o público-alvo é restringido aos iniciantes no nível superior. Além disso, a agregação dos resultados da RSL de Medeiros et al. (2013) e desta RSL foi feita para responder a uma nova Sub-Q visando evidenciar o que é consenso na literatura e o que é inovação:

- Sub-Q6: Quais os avanços obtidos no uso de jogos no ensino de programação para iniciantes em Computação no nível superior na última década?

\subsection{Estratégia de Pesquisa e Fontes de Busca}

A estratégia de pesquisa utilizada consistiu em buscas manuais nos anais dos últimos cinco anos (2013 a 2017) de quatro eventos e três revistas na área de Informática na Educação do Brasil. Além das fontes escolhidas por Medeiros et al. (2013) para 2008 a 2012, foram investigadas novas fontes que surgiram a partir de 2012, o que resultou na inclusão do evento DesafIE. Dessa forma, as buscas foram realizadas nas bases descritas a seguir: (1) Simpósio Brasileiro de Informática na Educação (SBIE); (2) Workshop de Desafios da Computação Aplicada à Educação (DesafIE); (3) Workshop de Informática na Escola (WIE); (4) Workshop sobre Educação em Computação (WEI); (5) Revista Brasileira de Informática na Educação (RBIE); (6) Revista de Informática Teórica e Aplicada (RITA); e (7) Revista Novas Tecnologias na Educação (RENOTE);

\subsection{Critérios de Seleção}

Todos os artigos escolhidos para esta RSL foram submetidos a um conjunto de critérios de seleção, conforme mostra a Tabela 3. Tais critérios foram adaptados de Medeiros et al. (2013) a fim de contemplarem somente os estudos referentes ao ensino no nível superior. A robótica educacional, discutida como uma ferramenta para auxiliar as novas metodologias de ensino-aprendizagem de programação, é relevante pelo seu caráter multidisciplinar [Attrot 2002]. Devido a isso, o seu uso foi incluído na análise desta RSL. O procedimento de seleção dos artigos foi feito em quatro passos, listados a seguir: (1) Leitura do título e do resumo; (2) Leitura da introdução e da conclusão; (3) Leitura do artigo completo; e (4) Revisão da atividade de seleção.

\subsection{Critérios de Qualidade}

Para avaliar os aspectos metodológicos dos estudos selecionados, foram utilizados os critérios de qualidade (CQ) levantados por Medeiros et al. (2013):

- CQ1: Foi utilizada alguma teoria pedagógica para auxiliar no processo de aprendizagem de programação?

- CQ2: Foi utilizada alguma ferramenta existente no mercado para auxiliar no processo de ensino-aprendizagem de programação?

- CQ3: Foi realizado algum tipo de experimento controlado ou estudo de caso real para avaliação da metodologia proposta? 
VII Congresso Brasileiro de Informática na Educação (CBIE 2018)

Anais do XXIX Simpósio Brasileiro de Informática na Educação (SBIE 2018)

- CQ4: O estudo avaliado apresenta uma breve comparação com outras metodologias relacionadas existentes?

- CQ5: O estudo avaliado apresenta o endereço (URL) na Internet onde o jogo digital utilizado esteja disponível para uso ou para cópia (download)?

- CQ6: O estudo avaliado foi aplicado em alguma universidade?

Tabela 3. Critérios de seleção dos artigos

\begin{tabular}{|c|c|c|}
\hline Tipo & ID & Critério \\
\hline Inclusão & CI1 & $\begin{array}{r}\text { O tema do artigo aborda o ensino de programação com jogos digitais e/ou robótica } \\
\text { para iniciantes em Computação no nível superior no Brasil. }\end{array}$ \\
\cline { 2 - 4 } & CI2 & $\begin{array}{r}\text { O tema do artigo é sobre o ensino de programação com jogos digitais e/ou robótica } \\
\text { para iniciantes em Computação no nível superior no Brasil. }\end{array}$ \\
\cline { 2 - 4 } & CI3 & A ferramenta utilizada no trabalho foi desenvolvida e está disponível. \\
\hline \multirow{3}{*}{ Exclusão } & CE1 & $\begin{array}{r}\text { O tema do artigo aborda o ensino de programação sem o uso de jogos digitais (e/ou } \\
\text { robótica) para iniciantes em Computação no nível superior no Brasil. }\end{array}$ \\
\cline { 2 - 4 } & CE2 & $\begin{array}{r}\text { O tema do artigo não é sobre o ensino de programação com jogos digitais (e/ou } \\
\text { robótica) para iniciantes em Computação no nível superior no Brasil. }\end{array}$ \\
\cline { 2 - 4 } & CE3 & A ferramenta utilizada no trabalho não foi desenvolvida. \\
\cline { 2 - 4 } & CE4 & Artigos duplicados. \\
\cline { 2 - 4 } & CE5 & Artigos resumidos. \\
\hline
\end{tabular}

A escala de pontuação dos critérios de qualidade utilizada foi proposta por Kitchenham e Charters (2007), baseando-se nas respostas: (1) Sim, 1 ponto; (2) Parcial, 0,5 ponto; e (3) Não, 0 ponto. Esta avaliação visa classificar a qualidade dos artigos, de modo que os estudos que obtiveram nota zero foram retirados da RSL. Nessa etapa, dois pesquisadores ( $\mathrm{P} 1$ e $\mathrm{P} 2$ ) leram individualmente os artigos selecionados e os pontuaram de acordo com a escala citada acima. Ao final, os resultados obtidos foram comparados por um terceiro pesquisador (P3), que julgou e resolveu divergências entre P1 e P2.

\subsection{Formulário de Extração de Dados}

Após a seleção, os artigos foram submetidos a uma extração sistemática com uso de um formulário definido visando responder as Sub-Qs. Isso favorece a síntese, pois permite a retirada uniforme de informações dos artigos, i.e., especifica dados a serem coletados de cada estudo incluído. Os campos que compõem esse formulário são: (1) estudo de caso real; (2) metodologia pedagógica; (3) ferramenta utilizada; (4) em que universidade foi desenvolvido o projeto; (5) resultado com os alunos; e (6) trabalhos futuros.

\section{Resultados}

A busca manual resultou em 34 artigos, dos quais 12 foram selecionados. Como esta revisão é uma replicação, foram incluídos ainda oito estudos de Medeiros et al. (2013), totalizando 20 para análise final. Os artigos reutilizados de 2013 atendem aos critérios exibidos nas Subseções 4.3 e 4.4. A Tabela 4 apresenta uma compilação do processo de 
VII Congresso Brasileiro de Informática na Educação (CBIE 2018)

Anais do XXIX Simpósio Brasileiro de Informática na Educação (SBIE 2018)

busca realizado nesta RSL referente aos anos 2008-2012 e 2013-2017 (atualização). Na Tabela 5, são apresentados os artigos selecionados e seus identificadores próprios (ID).

Tabela 4. Sumarização do processo de busca

\begin{tabular}{|c|c|c|c|c|c|c|}
\hline \multirow{2}{*}{ Evento/Revista } & \multicolumn{2}{|c|}{ Quantidade de Artigos } & \multicolumn{2}{c|}{ Busca Manual } & \multicolumn{2}{c|}{ Selecionados } \\
\cline { 2 - 7 } & $\mathbf{2 0 0 8 - 2 0 1 2}$ & $\mathbf{2 0 1 3 - 2 0 1 7}$ & $\mathbf{2 0 0 8 - 2 0 1 2}$ & $\mathbf{2 0 1 3 - 2 0 1 7}$ & $\mathbf{2 0 0 8 - 2 0 1 2}$ & $\mathbf{2 0 1 3 - 2 0 1 7}$ \\
\hline SBIE & 434 & 721 & 18 & 14 & 5 & 2 \\
\hline DesafIE & - & 69 & - & 0 & - & 0 \\
\hline WIE & 200 & 427 & 8 & 5 & 3 & 2 \\
\hline WEI & 122 & 215 & 13 & 10 & 0 & 5 \\
\hline RBIE & 96 & 156 & 4 & 1 & 0 & 1 \\
\hline RITA & 78 & 101 & 0 & 0 & 0 & 0 \\
\hline RENOTE & 584 & 570 & 3 & 4 & 0 & 2 \\
\hline
\end{tabular}

\subsection{Como os jogos digitais têm sido aplicados no ensino de programação?}

Para responder a Sub-Q1, compreender o nível de apoio (estudo de caso real) realizado por cada pesquisa é necessário para validar o processo de ensino-aprendizagem. Os artigos selecionados apresentam formas diferentes de avaliação: seis apresentaram oficinas como caso real (A1-2, A5, A9, A12, A19); dois recorreram a avaliações por especialistas (A3-4); sete foram realizados ao longo do período letivo dos cursos de Computação (A6-7, A13-16, A18); três utilizaram estudos experimentais com alunos (A8, A10-11); e dois ofereceram curso fora das disciplinas (A17, A20). A Figura 1 mostra um gráfico com a contagem dos casos reais. Pode-se perceber que a validação durante o período letivo é o método mais utilizado entre os estudos, enquanto as oficinas (validação do estudo em aula específica) ficaram em segundo lugar.

\subsection{Que ferramentas têm sido empregadas?}

Para responder a Sub-Q2, foram encontradas 15 ferramentas para apoio à programação: HTML, CSS, JavaScript, Adobe Flash Professional CS5, Blockly, Unity 3D, Construct 2, GameMaker, Lego Mindstorm, RoboMind, Stencyl, PyGame, Escracho, Alice e iVprog. HTML, CSS e Javascript são fundamentais para gerar documentos com padrões lidos por browsers (A1). Adobe Flash Professional CS5 é usado para criar conteúdo interativo e permitir manuseio de objetos 3D (A2). Blockly é uma biblioteca JavaScript da Google para construir editores de programação visual e se assemelha ao Scratch, usado como framework junto com HTML e Java (A5). Unity 3D é um motor de jogo multiplataforma que suporta animações 3D, tem licença proprietária e usa as linguagens $\mathrm{C}++$ e $\mathrm{C} \#$ (A3, A9). Construct 2 também é um motor que não requer codificação, com licença proprietária e tem suporte a Java e C++ (A8, A10). Outro motor, Game Maker, é proprietário, possui linguagem simples baseada em Delphi e conta com recursos próprios como editores de imagens, sons, scripts e fases (A16).

Por outro lado, Lego Mindstorms é uma linha do brinquedo LEGO que explora introdução à robótica, estimulando a criatividade e a solução de problemas (A6, A18). 
VII Congresso Brasileiro de Informática na Educação (CBIE 2018)

Anais do XXIX Simpósio Brasileiro de Informática na Educação (SBIE 2018)

RoboMind é uma linguagem de programação educacional para ensinar noções básicas de lógica, robótica e inteligência artificial em um ambiente simulador que, a partir de comandos do usuário, faz com que um robô explore um local (A13). Já Stencyl é uma ferramenta comercial para desenvolver jogos $2 \mathrm{D}$, cujos produtos podem ser exportados para Web via Adobe Flash Player (A4). PyGame é uma biblioteca de jogos com Python, com API em $\mathrm{C}$ e funcionalidades de programação gráfica, além de ter licença do tipo LGPL (A14). Escracho é um programa cuja linguagem contém elementos gráficos, que são arrastados e encaixados, desde que respeitem restrições sintáticas (A15). Alice é um ambiente de programação tridimensional com licença livre que possibilita o uso de animações 3D e arrastar instruções para criar jogos (A19). Por fim, o iVprog permite interação com elementos visuais, sendo usado para ensinar algoritmos em aplicações Web (A20). Alguns trabalhos não informaram a ferramenta usada (A7, A11-12, A17).

Tabela 5. Artigos selecionados para a RSL

\begin{tabular}{|c|c|c|c|c|}
\hline ID & Título & Ano & Instituição & Fonte \\
\hline A1 & Ensino de Algoritmos Apoiado pelo Uso de Jogos Digitais Educativos & 2013 & UDESC & RENOTE \\
\hline $\mathrm{A} 2$ & Jogo Baralho das Variáveis & 2013 & UFPA & WEI \\
\hline A3 & Teddy Racer: Lógica de Programação e Ludicidade & 2015 & IFES & RENOTE \\
\hline A4 & DEG4Trees: Um Jogo Educacional Digital de Apoio ao Ensino de Estruturas de Dados & 2015 & UFG & WEI \\
\hline A5 & Testando a Diversão em um Jogo Sério para o Aprendizado Introdutório de Programação & 2015 & UDESC & WEI \\
\hline A6 & Robótica Educativa na aprendizagem de Lógica de Programação: Aplicação e Análise & 2015 & IFBA & WIE \\
\hline A7 & $\begin{array}{c}\text { O Desafio da Serpente - Usando Gamification para Motivar Alunos em uma Disciplina } \\
\text { Introdutória de Programação }\end{array}$ & 2016 & UFPB & SBIE \\
\hline A8 & Um Modelo Lúdico para o Ensino de Conceitos de Programação de Computadores & 2016 & UNIFACS & SBIE \\
\hline A9 & POOGame: Um Jogo Sério para o Ensino de Programação Orientada a Objetos & 2016 & UFC & WEI \\
\hline A10 & Um Modelo Lúdico para o Ensino de Conceitos de Programação de Computadores & 2017 & UNIFACS & RBIE \\
\hline A11 & Game Logic: Um Jogo para Auxiliar na Aprendizagem de Lógica de Programação & 2017 & IFMS & WEI \\
\hline A12 & $\begin{array}{c}\text { Análise do Laboratório Remoto de Robótica Educacional (LERO) como Ferramenta de Ensino e } \\
\text { Aprendizagem de Introdução à Programação }\end{array}$ & 2017 & IFBA & WIE \\
\hline A13 & O uso do Lego Mindstorms no Apoio ao Ensino de Programação de Computadores & 2009 & FURB & WIE \\
\hline A14 & Aprendendo a Ensinar Programação Combinando Jogos e Python & 2010 & UFPB & SBIE \\
\hline A15 & Avaliação Empírica da Utilização de um Jogo para Auxiliar a Aprendizagem de Programação & 2010 & UNIVALI & SBIE \\
\hline A16 & ProGame: Um Jogo para o Ensino de Algoritmos e Programação & 2010 & IESP, UFPB & SBIE \\
\hline A17 & Uma Abordagem Lúdica para a Aprendizagem de Programação de Computadores & 2010 & PUC-PR & WIE \\
\hline A18 & $\begin{array}{c}\text { A Robótica como Ferramenta de Apoio ao Ensino de Disciplinas de Programação em Cursos de } \\
\text { Computação e Engenharia }\end{array}$ & 2011 & UFF & WIE \\
\hline A19 & Limitações da Utilização do Alice no Ensino de Programação para Alunos de Graduação & 2012 & PUC-PR & SBIE \\
\hline A20 & $\begin{array}{c}\text { Uma Visão do Cenário Nacional do Ensino de Algoritmos e Programação: Uma Proposta } \\
\text { Baseada no Paradigma de Programação Visual }\end{array}$ & 2012 & USP & SBIE \\
\hline
\end{tabular}


VII Congresso Brasileiro de Informática na Educação (CBIE 2018)

Anais do XXIX Simpósio Brasileiro de Informática na Educação (SBIE 2018)

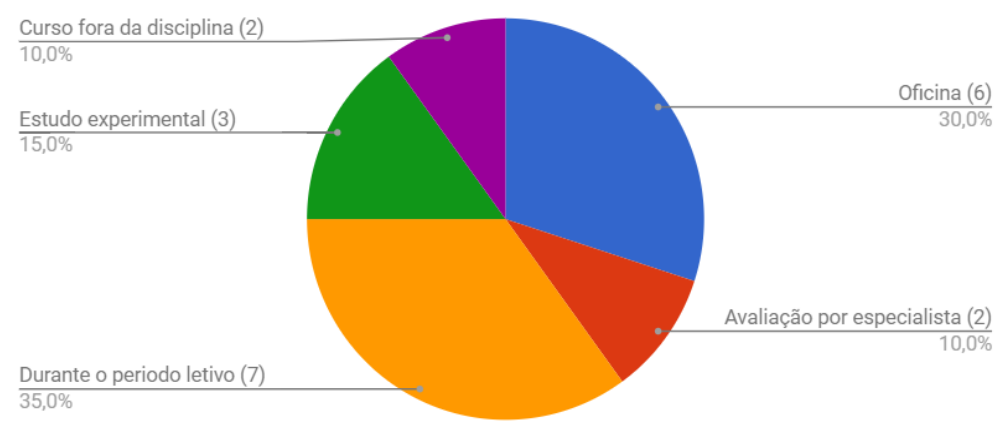

Figura 1. Contexto dos casos reais

\subsection{Quais os efeitos observados nos alunos com a introdução de atividades de programação baseadas em jogos digitais?}

A maioria dos estudos afirma que os jogos digitais utilizados no ensino de programação para iniciantes em Computação contribuíram para a aprendizagem. Um artigo não havia reportado avaliação (A7) e outro relatou que o uso de jogos digitais desenvolvidos com Alice não contribuiu para melhor rendimento do aluno em disciplinas de programação (A19). De modo geral, o uso de jogos digitais e/ou métodos lúdicos para o ensino de programação têm uma alta aceitação dos alunos, promovendo o seu engajamento.

\subsection{Que teorias pedagógicas estão sendo utilizadas?}

As principais teorias pedagógicas utilizadas pelos estudos são a Teoria Pedagógica do Construtivismo (CON) e a Taxionomia de Bloom (TX). CON tem como principais objetivos a construção do conhecimento e a autonomia do aprendiz, ou seja, propõe-se que o aluno participe ativamente do próprio aprendizado. Para Henrique e Silva (2017), os requisitos principais para CON são: (1) exibir feedback construtivo e individualizado; (2) propor reflexões críticas; (3) propor ao aprendiz construir a maior quantidade de soluções possíveis; e (4) permitir que realize escolhas durante a interação (A1, A6, A13, A18). Por sua vez, a TX (ou taxonomia dos objetivos educacionais) deve auxiliar a identificação da hierarquia do propósito do sistema ligado ao desenvolvimento cognitivo do aprendiz. Segundo Henrique e Silva (2017), os conteúdos do software educacional devem ser divididos em vários níveis de conhecimento, iniciando sempre do menor nível de complexidade (A7, A15-16). Treze artigos não explicitaram uso de teorias pedagógicas para desenvolver a metodologia de ensino de programação aos iniciantes em Computação no nível superior (A2-5, A8-12, A14, A17, A19, A20).

\subsection{Quais são as instituições de pesquisas envolvidas na área e como elas estão distribuídas no Brasil?}

Esta revisão encontrou 15 instituições realizando pesquisas na área. A Figura 2.a exibe a relação entre as instituições identificadas e a quantidade de trabalhos. Vale ressaltar que um dos estudos (A16) foi realizado pela IESP em conjunto com a UFPB. Além disso, pode-se perceber que a UFPB conduziu três estudos; UDESC, IFBA, UNIFACS e PUCPR conduziram dois; e as demais, apenas um. Na Figura 2.b, observa-se a distribuição das pesquisas em função das regiões. As regiões Nordeste, Sul e Sudeste são as que apresentam maior engajamento nas pesquisas na área, com $28,6 \%$ de autoria cada uma. 
(a)
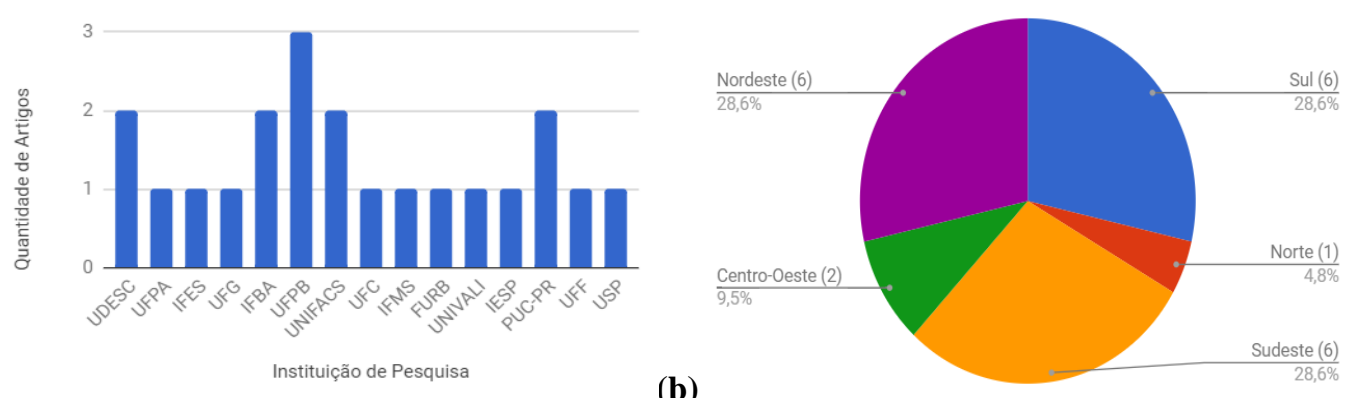

Figura 2. (a) Quantidade de artigos por instituição e (b) Percentual de artigos por região

\section{Discussão dos Resultados}

Visando responder a Sub-Q6, algumas observações são apontadas. A primeira se trata dos casos reais dos estudos. Nos últimos anos, as oficinas foram utilizadas de forma crescente, partindo de uma ocorrência no período 2008 a 2012 para cinco entre 2013 e 2017. Mesmo a quantidade de casos "durante o ano letivo" sendo maior, o seu uso decresceu com o passar dos anos, estando mais concentrado no primeiro quinquênio. Novas formas de avaliar os trabalhos surgiram com o passar dos anos, por exemplo, a avaliação por especialista e os estudos experimentais.

Dentre as ferramentas, Unity 3D, Construct 2 e Lego Mindstorm foram as mais utilizadas, sendo empregadas em dois estudos cada. A programação de desafios nesses ambientes é simples, o que pode ser uma das razões para o uso. A robótica educacional figurou em apenas três artigos, mas contribuiu para um aprendizado lúdico construtivo e motivador ao aprendiz, tornando-se um potencial instrumento de interesse no processo. Outro aspecto é o engajamento das regiões em pesquisas sobre jogos educacionais. As instituições no Nordeste apresentaram mais trabalhos em 2013-2017 em relação a 20082012. Há também trabalhos das regiões Centro-Oeste e Norte, que não constam entre os estudos do primeiro quinquênio. A região Sul apareceu com menos trabalhos em 20132017 e a região Sudeste se manteve ativa. Em relação aos anos das publicações, vale ressaltar que não foram encontrados trabalhos aderentes aos requisitos desta RSL nos anos 2008 e 2014 e que os anos 2010 e 2015 tiveram o maior número (quatro em cada).

Uma limitação enfrentada é a quantidade reduzida de artigos no tema desta RSL (20), pois o trabalho se restringiu ao uso de jogos digitais para ensino de programação aos iniciantes em Computação no nível superior no Brasil na última década. No entanto, os artigos analisados mencionaram esforços dos grupos para liberar novas versões dos jogos (com novas missões e mais aprendizes), bem como no acompanhamento dos alunos em novos experimentos. Observa-se pouco uso de metodologias pedagógicas, demonstrado pela quantidade de trabalhos que não explicitam quais técnicas utilizaram.

\section{Considerações Finais}

Este trabalho teve como objetivo apresentar uma RSL sobre o uso de jogos digitais no ensino de programação para iniciantes em Computação no nível superior na última década no Brasil, dado o potencial dos jogos no ensino-aprendizagem [Uzunca e Jansen, 2016]. A busca foi realizada em eventos e revistas nacionais e resultou em 20 artigos submetidos à extração uniforme de dados para mapear o cenário brasileiro. Pode-se identificar os principais requisitos e lacunas para a criação de jogos digitais para apoiar a aprendizagem construtivista e de qualidade. Conforme os resultados, essa nova forma 
VII Congresso Brasileiro de Informática na Educação (CBIE 2018)

Anais do XXIX Simpósio Brasileiro de Informática na Educação (SBIE 2018)

de se buscar aprendizado é eficaz quando empregada da maneira certa. Em torno de $90 \%$ dos artigos analisados alcançaram o seu propósito, ratificando a afirmação de Victal et al. (2015) e Hoelfmann (2016) sobre a sua importância como instrumento de apoio ao ensino. No entanto, o uso de jogos digitais ainda não é totalmente eficaz. Para Scaico e Scaico (2016), é preciso desenvolver estudos com maior rigor científico e metodológico. Devido à quantidade reduzida de trabalhos na área, percebe-se que o emprego de jogos digitais para o ensino requer mais pesquisas para agregar qualidades técnicas e reforçar a sua utilidade. Acredita-se que jogos digitais são ferramentas para o envolvimento e o aprendizado do aluno e o seu uso deve ser bem pensado, pois os jogos devem auxiliar o tutor no processo ensino-aprendizagem (e não o substituir). Como trabalho futuro, pretende-se investigar o cenário internacional e realizar análises mais abrangentes sobre a utilização de jogos digitais no ensino de programação.

Agradecimentos. O primeiro autor agradece o auxílio financeiro da UNIRIO.

\section{Referências}

Attrot, W., Ayrosa, P. (2002). "Aplicações da Robótica no Ensino de Ciência da Computação". In Anais do CSBC, WEI 2002.

Basili, V. (1992). "Software Modeling and Measurement: The Goal/Question/Metric Paradigm”. Technical Report CS-TR-2956, University of Maryland.

Domínguez, A. et al. (2013). "Gamifying learning experiences: Practical implications and outcomes". Computers \& Education 63(2013):380-392.

Henrique, M. e Silva, C. (2017). "Um catálogo de requisitos pedagógicos para auxiliar o desenvolvimento de Softwares Educacionais". In Anais do SBIE 2017, pp. 506-515.

Hoelfmann, C. (2016). "O uso dos jogos digitais educacionais no processo de ensino e aprendizagem". Universidade Federal de Santa Catarina.

Kapp, K. (2012). "The Gamification of learning and instruction: Game-based methods and strategies for training and education". Pfeiffer \& Company, $1^{a}$ edição.

Kitchenham, B., Charters, S. (2007). "Guidelines for Performing Systematic Literature Reviews in Software Engineering”. Keele Univ./Durham University Joint Report.

Medeiros, T. et al. (2013). "Ensino de programação utilizando jogos digitais: uma revisão sistemática da literatura". RENOTE 11(3):1-10.

Piteira, M., Haddad, S. (2011). "Innovate in your program computer class: an approach based on a serious game". In Proceedings of OSDOC 2011, pp. 49-54.

Santos, R. et al. (2008). "Uma Proposta de Cenário para Ensino de Algoritmos e Programação com Contribuições de Cooperação, Colaboração e Coordenação". In Anais do CSBC, WEI 2008, pp. 218-227.

Scaico, A., Scaico, P. (2016). "Uso de Jogos em Cursos Introdutórios de Programação no Ensino Superior na Área de Computação: Uma Revisão Sistemática”. In Anais do SBIE 2016, pp. 549-558.

Silva, T. et al. (2014). "Jogos Digitais para Ensino e Aprendizagem de Programação: uma Revisão Sistemática da Literatura”. In Anais do SBIE 2014, pp. 692-701.

Victal, E. et al. (2015). “Aprendendo sobre o uso de Jogos Digitais na Educação". In Anais do WIE 2015, pp. 444-453.

Uzunca, B., Jansen, S. (2016). "How do Ecosystem Dynamics work in Serious Gaming Ecosystems? Challenges and Opportunities". In Strategic Management Society. 